\title{
Hydrogen equilibration in polycrystalline silicon
}

\author{
K. von Maydell and N. H. Nickel ${ }^{\mathrm{a})}$ \\ Hahn-Meitner-Institut Berlin, Kekuléstr. 5, D-12489 Berlin, Germany
}

(Received 23 January 2007; accepted 19 February 2007; published online 29 March 2007)

\begin{abstract}
Hydrogen equilibration in polycrystalline silicon was investigated as a function of annealing time and temperature using electron spin resonance and hydrogen effusion measurements. During a vacuum anneal at least $1.5 \times 10^{21} \mathrm{~cm}^{-3} \mathrm{H}$ atoms are mobile in the lattice, however, only about $3.7 \times 10^{18} \mathrm{~cm}^{-3} \mathrm{H}$ atoms passivate $\mathrm{Si}$ dangling bonds. The results show that the annealing treatment can cause the vast majority of $\mathrm{H}$ atoms to accumulate in $\mathrm{H}$ stabilized platelets. Since defect passivation preferentially occurs at grain boundaries and platelet nucleation and growth are confined to the interior of single-crystal grains, $\mathrm{H}$ equilibration is governed by two spatially separated processes. Moreover, the data demonstrate that the hydrogen density-of-states distribution is dynamic and changes in response to experimental parameters. (C) 2007 American Institute of Physics. [DOI: 10.1063/1.2716348]
\end{abstract}

The properties of hydrogen in silicon have attracted a great deal of interest. ${ }^{1}$ This manifests itself in the neutralization of dopant atoms and the passivation of localized defects such as Si dangling bonds. The latter attribute is commonly utilized to improve the electrical and optical properties of polycrystalline silicon (poly-Si), which contains a high concentration of Si dangling bonds at grain boundaries. ${ }^{2}$ On the other hand, prolonged exposure of poly-Si to monatomic $\mathrm{H}$ can cause deleterious effects such as the formation of electrical and structural defects. 3,4

In poly-Si identical crystallites are separated by grain boundaries. These two-dimensional extended defects contain deep and shallow localized states. Posthydrogenation treatments at moderate temperatures (e.g., $250-450{ }^{\circ} \mathrm{C}$ ) reduce the concentration of both classes of localized states and the kinetics of the grain-boundary passivation are consistent with diffusion limited passivation. ${ }^{2}$ In addition to the passivation of localized states it has been shown that prolonged exposure of poly-Si causes deleterious effects such as the formation of acceptor-like states that leads to electrical-type conversion ${ }^{3}$ and the formation of hydrogen stabilized platelets. ${ }^{4}$ The latter effect is controlled by the Fermi energy.

In this letter, we present experimental evidence that merely the migration of hydrogen in poly-Si is sufficient to simultaneously cause beneficial and deleterious effects. A simple vacuum anneal results in a decrease of the $\mathrm{Si}$ dangling-bond concentration and the formation of large platelet-like structures. While the passivation occurs preferentially at grain boundaries the formation of platelets is confined to the interior of the grains. Hydrogen equilibration involves about $30 \%$ of all incorporated $\mathrm{H}$ atoms.

Polycrystalline silicon thin films were prepared by the following procedure. First, hydrogenated amorphous silicon thin films were grown by rf glow discharge decomposition of silane $\left(\mathrm{SiH}_{4}\right)$ at substrate temperatures ranging from 160 to $335^{\circ} \mathrm{C}$. Then the specimens were crystallized at room temperature and in high vacuum using the $308 \mathrm{~nm}$ line of an excimer laser and employing the step-by-step crystallization technique. ${ }^{6}$ The resulting poly-Si films revealed an average grain size of 100-200 $\mathrm{nm}$. Information on the defect

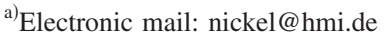

densities was obtained from electron spin resonance (ESR) measurements and the hydrogen density-of-states (H DOS) was deduced from $\mathrm{H}$ effusion data as described in Ref. 7.

Figure 1 shows ESR spectra of laser crystallized poly-Si before (blue spectrum) and after an additional vacuum anneal at $400{ }^{\circ} \mathrm{C}$ for $30 \mathrm{~min}$ (red spectrum). Both resonances exhibit a $g$ value of 2.0055 that is commonly attributed to $\mathrm{Si}$ dangling-bond defects. ${ }^{8}$ From the ESR spectra a Si danglingbond concentration of $N_{S}=6.2 \times 10^{18} \mathrm{~cm}^{-3}$ was estimated for as-crystallized poly-Si. This is a typical value for the spin density of completely crystallized poly-Si. ${ }^{9}$ A post-vacuumanneal at $T_{A}=400{ }^{\circ} \mathrm{C}$ for $30 \mathrm{~min}$ resulted in a decrease of the intensity of the $\mathrm{Si}$ dangling-bond resonance (red spectrum), and hence a decrease of the dangling-bond concentration to $N_{S}=3.8 \times 10^{18} \mathrm{~cm}^{-3}$.

The time and temperature dependence of $N_{S}$ is shown in Fig. 2. For all poly-Si specimens prepared by laser crystallization of hydrogenated amorphous silicon $(a-\mathrm{Si}: \mathrm{H})$ a decrease of the Si dangling-bond density is observed after a post-vacuum-anneal (triangles, squares, and circles in Fig. 2). With increasing annealing temperature $T_{A}$, the time to minimize $N_{S}$ decreases and at long annealing times $t_{A}$, the $\mathrm{Si}$

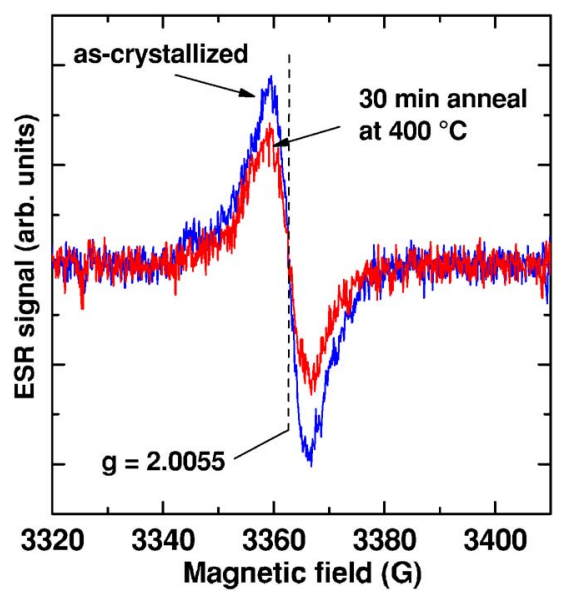

FIG. 1. (Color online) Electron spin resonance spectra of polycrystalline silicon at $300 \mathrm{~K}$. The blue spectrum shows the silicon dangling-bond resonance after laser crystallization. The spectrum shown by the red curve was obtained after the sample was subjected to a post-annealing treatment for $30 \mathrm{~min}$ at $400{ }^{\circ} \mathrm{C}$ and in vacuum. 


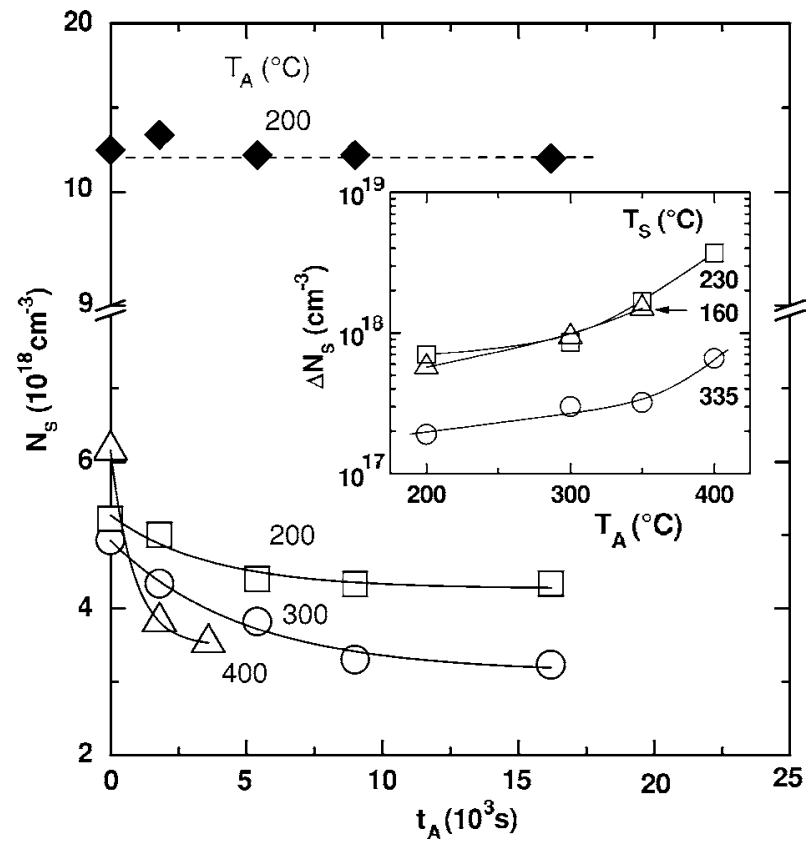

FIG. 2. Density of silicon dangling bonds, $N_{S}$, vs annealing time $t_{A}$ for various indicated annealing temperatures $T_{A}$. The triangles, squares, and circles represent data that were obtained from poly-Si where the initial material was hydrogenated amorphous silicon. The diamonds represent data where the initial amorphous Si film was grown by sputter deposition. The solid lines are fits to an exponential decay. The inset shows the total change of the Si dangling-bond concentration, $\Delta N_{S}$, as a function of the annealing temperature $T_{A} . T_{S}$ denotes the substrate temperature at which the $a-\mathrm{Si}: \mathrm{H}$ starting material was deposited.

dangling-bond concentration saturates. Moreover, with increasing annealing temperature the number of additionally passivated Si dangling bonds increases from $9 \times 10^{17} \mathrm{~cm}^{-3}$ at $T_{A}=200{ }^{\circ} \mathrm{C}$ to $2.7 \times 10^{18} \mathrm{~cm}^{-3}$ at $T_{A}=400{ }^{\circ} \mathrm{C}$.

The observed decrease of $N_{S}$ can be due to either structural changes at the grain boundaries or the activation of residual hydrogen leading to the formation of additional $\mathrm{Si}-\mathrm{H}$ complexes. To clarify which of these two processes is responsible for the defect passivation during post-vacuumanneals, poly-Si specimens were prepared from sputter deposited amorphous silicon. It is important to note that these samples contain a hydrogen concentration of less than 0.1 at. \%. After crystallization the samples exhibit a dangling-bond concentration of $N_{S}=1.3 \times 10^{19} \mathrm{~cm}^{-3}$. This value is about a factor of 3 larger than for samples where the starting material was hydrogenated amorphous silicon. A subsequent vacuum anneal for up to $16200 \mathrm{~s}$ did not result in the reduction of the dangling-bond concentration (full diamonds in Fig. 2). This clearly demonstrates that the observed decrease of $N_{S}$ is due to the activation of residual $\mathrm{H}$ and the subsequent passivation of Si dangling bonds.

The inset of Fig. 2 shows the total change of the $\mathrm{Si}$ dangling-bond concentration, $\Delta N_{S}$, as a function of the annealing temperature for poly-Si samples where the amorphous starting material was deposited at the indicated substrate temperatures $T_{S}$. Independent of the deposition temperature of the starting material the number of passivated Si dangling bonds increases with increasing $T_{A}$. The largest value of $\Delta N_{S}=3.7 \times 10^{18} \mathrm{~cm}^{-3}$ is obtained at an annealing temperature of $400{ }^{\circ} \mathrm{C}$ for poly-Si where the $a-\mathrm{Si}: \mathrm{H}$ starting material was deposited at $T_{S}=230{ }^{\circ} \mathrm{C}$. When the starting material is deposited at higher substrate temperatures (circles in

Downloaded 16 Jul 2007 to 134.30 .4 .55. Redistribution subject
Fig. 3) the decrease of the Si dangling-bond concentration is about a factor of 4-6 smaller than in specimens deposited at $T_{S} \leqslant 230^{\circ} \mathrm{C}$. Most likely, this is due to the fact that the $\mathrm{H}$ content of the amorphous starting material decreases with increasing substrate temperature. ${ }^{10}$

To elucidate the underlying microscopic mechanisms the hydrogen density-of-states distribution was derived from $\mathrm{H}$ effusion measurements. The H DOS of "as-crystallized" poly-Si and after different post-crystallization treatments are shown in Fig. 3. The energy scale was adjusted to a relative scale where the zero of energy corresponds to a free $\mathrm{H}$ atom. Also shown are the energies per $\mathrm{H}$ atom of a number of $\mathrm{H}$ complexes in crystalline silicon $(c-\mathrm{Si})$ according to Refs. 11 and 12. The influence of a post-vacuum-anneal of poly-Si at $T_{A}=350{ }^{\circ} \mathrm{C}$ for $2.5 \mathrm{~h}$ on the $\mathrm{H}$ DOS is shown in Fig. 3(a). The as-crystallized poly-Si sample exhibits a broad hydrogen distribution between $E=-2.5$ and $-3.7 \mathrm{eV}$ (red curve). However, a simple post-vacuum-anneal causes a spectacular change of the H DOS, namely, the formation of a pronounced peak located at $E=-2.9 \mathrm{eV}$ (blue curve). By comparing the position of the peak to the calculated $\mathrm{H}$ binding energies of hydrogen complexes in $c$-Si it can be assigned to $\mathrm{H}$ stabilized platelets. This is in accordance with a previous report, which showed that platelets form preferentially in undoped and $n$-type poly-Si. ${ }^{4}$

Further insight into the passivation mechanism was obtained by comparing the influence of the vacuum anneals on as-crystallized to post-hydrogenated poly-Si. Therefore, a laser crystallized specimen was exposed to monatomic $\mathrm{H}$ in an optically isolated remote plasma system for $1 \mathrm{~h}$ at $350{ }^{\circ} \mathrm{C}$. Next, one-half of the sample was annealed in vacuum for $2.5 \mathrm{~h}$ at $T_{A}=350{ }^{\circ} \mathrm{C}$. Then the $\mathrm{H}$ DOS was derived from $\mathrm{H}$ effusion measurements [red curve in Fig. 3(b)]. Posthydrogenation did not alter the shape of the H DOS, however, the total $\mathrm{H}$ concentration increased, which is consistent with previous results. ${ }^{2,13,14}$ On the other hand, the additional vacuum anneal also resulted in the formation of a pronounced peak located at $E=-3.04 \mathrm{eV}$. The energetic position of the peak suggests that it also originates from $\mathrm{H}$ stabilized platelets. Analyzing the area under the two peaks reveals that about $1.5 \times 10^{21} \mathrm{~cm}^{-3} \mathrm{H}$ atoms are accommodated in platelets after the vacuum anneal at $T_{A}=350{ }^{\circ} \mathrm{C}$.

The experimental data clearly show that hydrogen equilibration is governed by two distinctively different mechanisms, namely, the passivation of Si dangling bonds and the formation of platelets. Both processes occur simultaneously and are driven by $\mathrm{H}$ motion in the host lattice. According to the data shown in Fig. 3 the samples contain about $5 \times 10^{21} \mathrm{~cm}^{-3} \mathrm{H}$ atoms. After the vacuum anneals, at least $30 \%$ of the $\mathrm{H}$ atoms have migrated in the specimens. However, only a small fraction of about $3.7 \times 10^{18} \mathrm{~cm}^{-3} \mathrm{H}$ atoms have passivated additional Si dangling bonds. The ratio of $\mathrm{H}$ atoms passivating grain-boundary defects to $\mathrm{H}$ atoms accommodated in platelets is a measure for the passivation efficiency $\eta$. For postannealing at $T_{A}=350^{\circ} \mathrm{C}$ a passivation efficiency of $\eta \approx 2.5 \times 10^{-3}$ is obtained. This indicates that the migration of about $400 \mathrm{H}$ atoms is required to passivate one Si dangling bond. For plasma passivation of poly-Si a much larger passivation efficiency of $\eta \approx 0.055$ is observed. $^{2}$

The data show that most hydrogen atoms are accommodated in poly-Si without passivating preexisting defects. However, this requires that $\mathrm{H}$ be accommodated as diatomic AIP license or copyright, see http://apl.aip.org/apl/copyright.jsp 


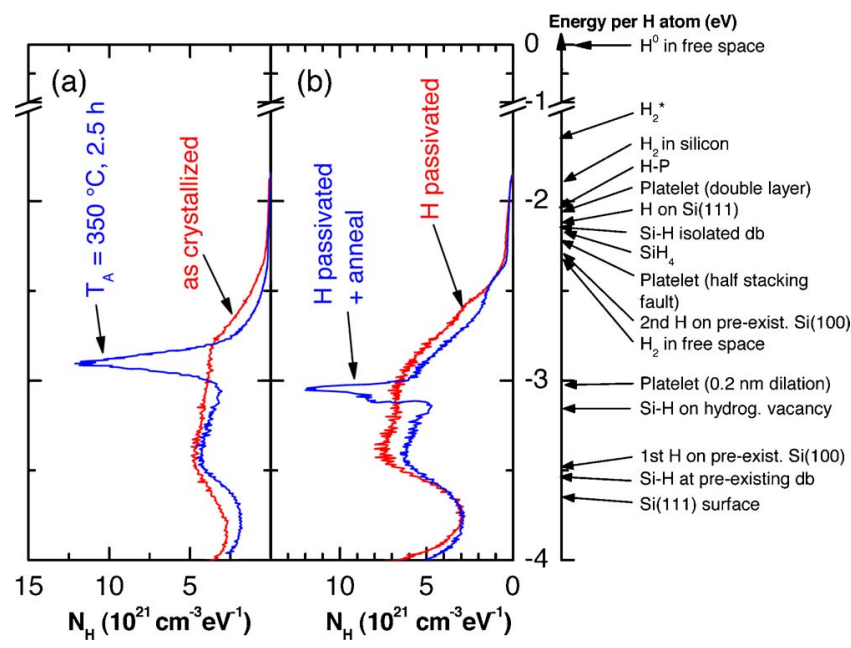

FIG. 3. (Color online) Hydrogen density-of-states distribution of laser crystallized poly-Si. The data plotted in (a) show the influence of a postvacuum-anneal at $T_{A}=350{ }^{\circ} \mathrm{C}$ for $2.5 \mathrm{~h}$ on the $\mathrm{H}$ DOS while (b) shows the effect of post-hydrogenation at $350{ }^{\circ} \mathrm{C}$ for $1 \mathrm{~h}$ followed by a vacuum anneal at $T_{A}=350^{\circ} \mathrm{C}$ for $2.5 \mathrm{~h}$. The data are adjusted to a relative energy scale where zero energy corresponds to a free $\mathrm{H}$ atom. Calculated energies of $\mathrm{H}$ complexes in single-crystal silicon are shown (Refs. 11 and 12).

complexes. Possible sites include strained $\mathrm{Si}-\mathrm{Si}$ bonds near grain boundaries and interstitial molecular $\mathrm{H}_{2}$. The passivation of strained $\mathrm{Si}-\mathrm{Si}$ bonds occurs by the formation of $\mathrm{H}_{2}^{*}$ complexes. These complexes consist of one $\mathrm{H}$ in a $\mathrm{Si}-\mathrm{Si}$ bond center site forming a $\mathrm{Si}-\mathrm{H}$ band and the second $\mathrm{H}$ residing in the antibonding interstitial site forming a second $\mathrm{Si}-\mathrm{H}$ bond. ${ }^{15}$ Since the main constituents of poly-Si are crystalline grains and grain boundaries, the difference in passivation efficiency must be related to the binding energy of $\mathrm{H}$ in the reservoir. While $\eta$ is significantly smaller after laser crystallization the binding energies of $\mathrm{H}$ in the reservoir must be larger than after plasma passivation. This is consistent with the binding energies for $\mathrm{H}_{2}^{*}$ and $\mathrm{H}_{2}$ that amount to -1.65 and $-1.92 \mathrm{eV}$, respectively. Hence, it is conceivable that laser crystallization of $a-\mathrm{Si}: \mathrm{H}$ causes the formation of molecular $\mathrm{H}_{2}$ while posthydrogenation preferably leads to the creation of $\mathrm{H}_{2}^{*}$ complexes.

Although defect passivation and platelet formation happen simultaneously, both effects occur spatially separated. ESR experiments performed on doped poly-Si showed that dangling bonds are predominantly located at the grain boundaries. ${ }^{16}$ On the other hand, a detailed investigation on the formation of platelets in poly-Si revealed that these $\mathrm{H}$ stabilized two-dimensional structures form only in the interior of the grains. Moreover, the presence of lattice strain in the form of grain boundaries inhibits the formation of platelets. ${ }^{4}$ Thus, our data clearly show that $\mathrm{H}$ equilibration is governed by two spatially separated processes. Moreover, the data in Fig. 3 clearly show that the H DOS is dynamic. Hydrogen creates its own traps preferably by forming multihydrogen clusters because of their low entropy. Since a considerable concentration of $\mathrm{H}$ atoms is required to account for the peak in the H DOS, it can be concluded that the formation of $\mathrm{H}$ stabilized platelets requires long range hydrogen motion.

In summary, we have presented direct experimental evidence that hydrogen equilibration in poly-Si is governed by two spatially separated processes, namely, defect passivation at grain boundaries and the formation of platelets within the grains. $\mathrm{H}$ equilibration involves about $1.5 \times 10^{21} \mathrm{~cm}^{-3} \mathrm{H}$ atoms of which only about $3.7 \times 10^{18} \mathrm{~cm}^{-3} \mathrm{H}$ atoms passivated $\mathrm{Si}$ dangling bonds. The vast majority of $\mathrm{H}$ atoms are accommodated in platelets. Moreover, the data clearly show that the H DOS is dynamic and changes in response to experimental parameters.

${ }^{1}$ N. H. Nickel, Hydrogen in Semiconductors II (Academic, San Diego, 1999), Vol. 1.

${ }^{2}$ N. H. Nickel, N. M. Johnson, and W. B. Jackson, Appl. Phys. Lett. 62, 3285 (1993).

${ }^{3}$ N. H. Nickel, N. M. Johnson, and J. Walker, Phys. Rev. Lett. 75, 3720 (1995).

${ }^{4}$ N. H. Nickel, G. B. Anderson, and J. Walker, Solid State Commun. 99, 427 (1996).

${ }^{5}$ N. H. Nickel, G. B. Anderson, N. M. Johnson, and J. Walker, Phys. Rev. B 62, 8012 (2000).

${ }^{6}$ P. Lengsfeld, N. H. Nickel, and W. Fuhs, Appl. Phys. Lett. 76, 1680 (2000).

${ }^{7}$ N. H. Nickel and K. Brendel, Phys. Rev. B 68, 193303 (2003).

${ }^{8}$ N. M. Johnson, D. K. Biegelsen, and M. D. Moyer, Appl. Phys. Lett. 40, 882 (1982).

${ }^{9}$ P. Lengsfeld and N. H. Nickel, in Laser Crystallization of Silicon, edited by N. H. Nickel (Elsevier Academic, Paris, 2003), Vol. 75, 119.

${ }^{10} \mathrm{~W}$. Beyer, in Hydrogen in Semiconductors II, edited by N. H. Nickel (Academic, San Diego, 1999), Vol. 61, 165.

${ }^{11}$ C. G. Van de Walle, Phys. Rev. B 49, 4579 (1994).

${ }^{12}$ W. B. Jackson, Curr. Opin. Solid State Mater. Sci. 1, 562 (1996).

${ }^{13}$ N. H. Nickel and I. E. Beckers, Phys. Rev. B 66, 075211 (2002).

${ }^{14}$ N. H. Nickel, W. B. Jackson, and J. Walker, Phys. Rev. B 53, 7750 (1996).

${ }^{15}$ K. J. Chang and D. J. Chadi, Phys. Rev. Lett. 62, 937 (1989).

${ }^{16}$ K. Brendel, N. H. Nickel, K. Lips, and W. Fuhs, J. Non-Cryst. Solids 338-340, 262 (2004). 